Capacitance and conductance of mesoscopic systems connected by quantum point contacts

Flensberg, Karsten

Published in:

Physical Review B

DOI:

10.1103/PhysRevB.48.11156

Publication date:

1993

Document version

Early version, also known as pre-print

Citation for published version (APA):

Flensberg, K. (1993). Capacitance and conductance of mesoscopic systems connected by quantum point contacts. Physical Review B, 48(15), 11156-11166. https://doi.org/10.1103/PhysRevB.48.11156 


\title{
Capacitance and conductance of mesoscopic systems connected by quantum point contacts
}

\author{
Karsten Flensberg \\ Nordita, Blegdamsvej 17, 2100 Copenhagen $\varnothing$, Denmark
}

(Received 20 October 1992; revised manuscript received 18 June 1993)

\begin{abstract}
We study the transport properties of quantum dots and quantum point contacts in the Coulomb blockade regime and in the limit where the quantum point contact has nearly fully transmitting channels. Using a transformation to a multichannel Tomonaga-Luttinger-type model, we find the scaling behavior of the junction close to pinchoff. It is shown that the junction scales to an insulating junction. We find a crossover between a low-temperature regime with Coulomb blockade to a hightemperature regime where quantum charge fluctuations are dominant. The crossover temperature between these regimes is given by $T_{c} \sim U\left[1-G_{0} / N G_{H}\right]^{N / 2}$, where $U$ are the bare charging energy, $G_{0}$ is the nominal conductance, $N$ is the number of channels, and $G_{H}=e^{2} / h$.
\end{abstract}

\section{INTRODUCTION}

Since the work by Landauer ${ }^{1}$ our understanding of transport in mesoscopic systems has been guided by the relation between the conductance and the transmission amplitude, expressed in the Landauer formula. The Landauer formula is, however, not sufficient to describe the conductance of mesoscopic systems, such as small capacitance junctions and dots where correlation effects are important. In recent years, it has become experimentally achievable to fabricate microstructures small enough to observe the blocking of the tunneling current due to the charging energy associated with a single electron, an effect known as the Coulomb blockade. ${ }^{2}$ Small capacitance quantum dots (QD's) is an example of such systems where single electron charging effects are observable, ${ }^{3-8}$ leading to the so-called Coulomb oscillations in the tunneling current through the island. These developments have made it necessary to understand the role of quantum charge fluctuations which is an inherent feature of these devices. One source of charge fluctuations is the electro-magnetic environment of the junction. ${ }^{9-11}$ More interestingly, charge fluctuations within the mesoscopic sample itself may lead to a smearing of correlation effects. The transparency of the sample will determine the reduction of the charging energies and hence the observed capacitance of a mesoscopic sample is, like the conductance, related to its transmission amplitude.

Experimental evidence for the transmission amplitude dependence of the observed capacitance has been given by Kouwenhoven et al. ${ }^{4}$ who observed that the Coulomb oscillation of the conductance through a quantum dot confined between two quantum point contacts (QPC's) vanishes as the conductance of one of the QPC's approaches the value $2 G_{H}$. A similar effect is seen by Foxman et al. ${ }^{5}$

Also in tunnel junctions with a large number of transverse channels does the departure from non-Ohmic behavior depend strongly on the nominal conductance of the junction. ${ }^{12,13}$ There have been both experimental ${ }^{12,13}$ and theoretical ${ }^{14-18}$ attempts to address the question of how the Coulomb blockade is modified by charge transfer fluctuations. Odintsov ${ }^{14}$ and later Zwerger and Scharpf ${ }^{17}$ showed theoretically that only at temperatures $T<\exp \left(-G_{0} / 2 G_{H}\right)$ do the tunnel junctions exhibit blocking of the tunnel current. We find similar results in the case of a few nearly fully transmitting channels.

In this paper, we calculate the conductance of QD devices in the limiting case where the connecting QPC's have channels with transmission amplitudes close to 1 . This is done by mapping the model Hamiltonian to a series of coupled one-dimensional Tomonaga-type models. Inspired by the recent paper by Kane and Fisher, ${ }^{19}$ the model Hamiltonian is considered in two representations where the junction is viewed as a weak link or a weak barrier, respectively. In the latter case, we find an effective action, which involves only the local charge on the tunnel junction which is then used to calculate the conductance. It is found that the Coulomb blockade is strongly modified by the charge transfer fluctuations through the QPC. A crossover temperature between an Ohmic regime and a regime with Coulomb blockade is identified. The crossover temperature goes to zero as the reflection probability of the QPC goes to zero.

The paper is organized as follows: In Sec. II, we introduce the model, which in Sec. III is transformed to the bosonized form and an effective action is derived. This action is used in Sec. IV to calculate the conductance. In Sec. $\mathrm{V}$ we analyze the effective action by scaling arguments. Section VI deals with the predictions for the measured conductance and capacitance, and a discussion of the results is in Sec. VII.

\section{THE MODEL HAMILTONIAN}

First we briefly discuss the experimental configurations that are being considered. ${ }^{3-6,8}$ Figure 1 shows a typical geometry of a QD formed by electrostatic confinement of a two-dimensional electron gas (2DEG) in 


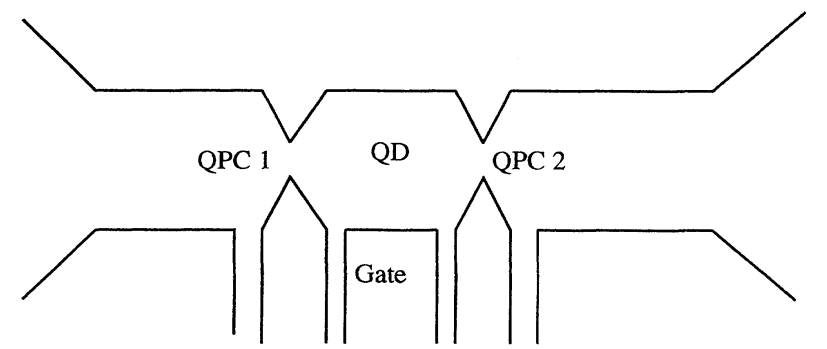

FIG. 1. Typical geometry of the quantum dot experiments discussed in this paper. The dot is connected to leads by two quantum point contacts. Their widths can be controlled by the gates as can the potential of the dot. We study the case where the quantum point contact has a conductance close to the first quantized value. The number of channels with transmission close to 1 is $N_{1}$ and $N_{2}$, respectively.

a GaAs $/ \mathrm{Al}_{x} \mathrm{Ga}_{1-x} \mathrm{As}$ heterostructure. The electrostatic potential is set up by the gates. Typical size of the QD is $500 \mathrm{~nm}$, and it contains of the order of one hundred electrons. The mean free path due to impurity scattering is much larger than the dimensions of the device. The QD is connected to the 2DEG through QPC's.

Next we discuss the modeling of this system. If the confining potential of the QPC is sufficiently smooth (which is a reasonable approximation because of the electrostatic nature of the confining potential) the single particle states can be described in the adiabatic picture. ${ }^{20}$ Under this condition the electronic states are quasi-onedimensional and the single electron part of the Hamiltonian can be approximated as

$$
H_{\mathrm{el}}=H_{0}+H_{\mathrm{barr}}
$$

where

$$
H_{0}=\sum_{n=1}^{N} \int d x \Psi_{n}^{\dagger}(x) \frac{p_{x}^{2}}{2 m} \Psi_{n}(x),
$$

and the barrier is described by

$$
H_{\mathrm{barr}}=\sum_{n=1}^{N} \int d x V_{n}(x) \Psi_{n}^{\dagger}(x) \Psi_{n}(x) .
$$

Here we have defined the field operators $\Psi_{n}(x)=$ $\int d y \phi_{n x}(y) \Psi(x, y)$, where $\phi_{n x}(y)$ is the eigenfunction for the transverse direction. $N$ is the number of channels included. The transverse eigenstates depend parametrically on $x$. The adiabatic picture breaks down as the contact becomes wider and the individual channels start to mix. Because there are a large number of states in the dot the electron transport from one QPC to the other is assumed to be incoherent and we can treat the two contacts independently. (In the case of an applied strong magnetic field adiabatic channels that connect the two contacts can exist. ${ }^{6}$ ) It is thus assumed that the thermal energy is larger than the level spacing energy in the dot.

Next we discuss the role of the electron-electron interactions in the quantum dot geometry shown in Fig. 1. The electron-electron interactions in the tunnel barrier

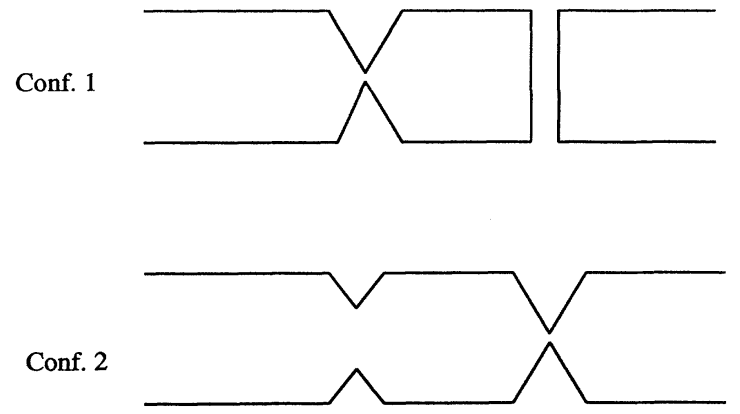

FIG. 2. The configurations of the device in Fig. 1 that is considered in the paper. In both cases $G_{2}<G_{1}$. Configuration 1: contact 2 is in the tunneling regime whereas contact 1 has channels with small reflection probability. Second configuration: contact 2 has channels close to pinchoff and contact 1 has a number of fully transmitting channels. See Table I for a summary of results.

region are neglected because of the low density of electrons there. It is furthermore assumed that a large number of transverse modes are occupied in the leads as well as in the dot such that the electron-electron interaction is well screened and we can view the $\mathrm{QD}$ as a capacitor. In terms of time scales this involves the approximation that the rearrangement of the charge distribution in the dot happens on a time scale (typical of order of the inverse plasma frequency in the dot) much faster than the characteristic time of the tunnel event, $\tau_{D}=\hbar /\left(e^{2} / 2 C_{D}\right)$, where $C_{D}$ is the total capacitance of the dot (dot-to-lead and dot-to-gates). With these approximations we can write the interaction energy as simply the electrostatic energy

$$
H_{\text {int }}=U_{D}\left(q_{1}+q_{2}\right)^{2}
$$

where $U_{D}=e^{2} / C_{D}$, and $q_{i}$ is the number of electrons that have passed through contact number $i$ into the dot, relative to the equilibrium number of electrons in the dot. In summary, the assumptions that justified the electrostatic model for the electron-electron interactions are (i) we must require a large density of states in the dot and in the leads, that is, the level spacing is small compared to the relevant energy scales for the experiment, and (ii) interactions in the contacts and in the leads can be neglected. This requirement is somewhat questionable in the case of several open and nearly open channels in the QPC's. However, when the width of the contact is small compared to the dot size, the traversal time for the passage through the QPC is much smaller than $\tau_{D}$ and hence the transfer of charge from the leads to the dot is governed by the correlation energy in the dot. The electrostatic energy model is thus expected to be valid also in that case if the width of the point contact is much smaller than $\hbar v_{F} / U_{D}$, which is typically the case. In this connection it is interesting to note that Coulomb oscillation effects have also been observed when the contacts have fully transmitting channels. ${ }^{7,8}$

The total model Hamiltonian now reads

$$
H=H_{1 \mathrm{el}}+H_{2 \mathrm{el}}+H_{\text {int }},
$$


where $H_{i \text { el }}$ describes the single electron part of junction number $i$. As explained above we neglect simultaneous tunneling (cotunneling) through both contacts which are thus treated independently. It was shown by Averin and Nazarov $^{21}$ that the cotunneling contribution to the linear conductance is proportional to the level spacing in the dot which we have assumed to be the smallest energy in our system. Therefore, in our model tunnel events only couple through the interaction term. Along similar lines of reasoning we can assume that once an electron has passed through the constriction the probability of being scattered back through the contact opening is negligible. This allows us to treat the adiabatic modes as onedimensional channels with scattering taking place solely at the constrictions.

The conductance of the device discussed above will be calculated in two simplifying limits, see Fig. 2. For the first configuration it is assumed that $G_{2} \ll G_{1}, G_{H}$ such that we can assume that the dot is in equilibrium with lead 1. (Here $G_{i}$ is the nominal conductance of QPC number $i$.) The conductance of the device can therefore be calculated with the usual tunneling theory to second order in the tunneling matrix elements. With these assumptions we get the standard expression for tunneling current,

$$
\begin{aligned}
I= & \frac{G_{2}}{e} \int \frac{d \omega}{2 \pi}\left[(\omega-e V) n_{\mathrm{B}}(\omega-e V)\right. \\
& \left.-(\omega+e V) n_{\mathrm{B}}(\omega+e V)\right] P(\omega),
\end{aligned}
$$

where $n_{\mathrm{B}}$ is the Bose function, $e V$ is the voltage difference across contact number 2 , and $P(\omega)$ is a spectral function defined by

$$
P(\omega)=\int_{-\infty}^{\infty} d t e^{i \omega t} P(t)
$$

where

$$
\begin{aligned}
P(t) & =\left\langle e^{i\left(H_{1 \mathrm{el}}+U_{D} q_{1}^{2}\right) t} e^{-i\left[H_{1 \mathrm{el}}+U_{D}\left(q_{1}+1\right)^{2}\right] t}\right\rangle_{1} \\
& =\left\langle T \exp \left(-i \int_{0}^{t} d t^{\prime} \hat{q}_{1}\left(t^{\prime}\right)\right)\right\rangle_{1} e^{-i U_{D} t} .
\end{aligned}
$$

The function $P$ is in general a complicated many-body object. However, under the assumption that the charging energy is small compared with the bandwidths of the channels connecting the dot to the lead, we can formulate the problem in terms of a Tomonaga-Luttinger-type model. This will be done in the subsequent section.

The second configuration considered is the case where $G_{2}$ is close to pinchoff and contact 1 has a number of transmitting channels. For this configuration we cannot use perturbation theory in the tunneling term but instead we will calculate the conductance using perturbation theory in the scattering barrier.

\section{BOSONIZATION AND EFFECTIVE ACTION}

\section{A. Boson form of the electron Hamiltonian}

As explained in Sec. II, the QPC may be regarded as a a series of one-dimensional problems, one for each channel, which are then coupled through the charging energy term. Quantization of the one-dimensional states in a box of size $L$ gives

$$
\begin{aligned}
H_{0} & =\sum_{n=1}^{N} \sum_{k} \epsilon_{n k} c_{n k}^{\dagger} c_{n k}, \\
H_{\text {barr }} & =\sum_{n=1}^{N} \frac{V_{n}}{L} \sum_{k k^{\prime}} c_{n k}^{\dagger} c_{n k^{\prime}}
\end{aligned}
$$

where we have modeled the barriers by $\delta$-function potentials: $V_{n}(x)=V_{n} \delta(x)$, since the detailed shape of the potential is not important for the low-energy behavior. The one-dimensional nature allows us to transform the problem to a coupled set of Tomonaga- or Luttinger-type models. ${ }^{22,23}$ The basic approximation in this model is the linearization of the electron energies,

$$
\epsilon_{n k}=v_{F n}\left(|k|-k_{F n}\right) .
$$

In the Luttinger model it is furthermore assumed that the dispersion curve does not terminate at $k=0, k_{D}$, where $k_{D}$ is the bandwidth, but continues to $\pm \infty$. The thus unphysical states introduced do not alter the results as long as effects of the finite bandwidth are unimportant. In the present case we are interested in the transport properties at low energies compared to the bandwidths, and for simplicity we therefore adopt the Luttinger model.

Here the basic steps are briefly reviewed. The bosonization starts with defining the density operators,

$$
\begin{aligned}
& \rho_{n i}(k)=\sum_{k^{\prime}} c_{n k^{\prime} i}^{\dagger} c_{n\left(k^{\prime}+k\right) i}, \\
& \rho_{n i}(-k)=\sum_{k^{\prime}} c_{n\left(k^{\prime}+k\right) i}^{\dagger} c_{n k^{\prime} i},
\end{aligned}
$$

where $k>0$. The density operators obey the commutation relations,

$$
\begin{aligned}
& {\left[\rho_{n 1}(-k), \rho_{n^{\prime} 1}\left(k^{\prime}\right)\right]=\left[\rho_{n 2}\left(k^{\prime}\right), \rho_{n^{\prime} 2}(-k)\right]=\frac{k L}{2 \pi} \delta_{n n^{\prime}} \delta_{k k^{\prime}},} \\
& {\left[\rho_{n 2}(-k), \rho_{n^{\prime} 1}\left(k^{\prime}\right)\right]=0 .}
\end{aligned}
$$

Physically, the $c_{1}$ and $c_{2}$ operators correspond to particles moving to the left and to the right, i.e., the electrons have been separated into two sections corresponding to positive and negative $k$ 's. If we write the unperturbed part of the Hamiltonian as

$$
H_{0}=\sum_{n=1}^{N} \sum_{i=1}^{2} \sum_{k} \epsilon_{n k} c_{n k i}^{\dagger} c_{n k i},
$$

we have the commutation relations,

$$
\begin{aligned}
& {\left[H_{0}, \rho_{n 1}(k)\right]=k \rho_{n 1}(k),} \\
& {\left[H_{0}, \rho_{n 2}(k)\right]=-k \rho_{n 2}(k) .}
\end{aligned}
$$

In the standard way $H_{0}$ can now be written in terms of the density operators,

$$
\begin{aligned}
H_{0} & =\frac{2 \pi}{L} \sum_{n=1}^{N} v_{F n} \sum_{k>0}\left[\rho_{n 1}(k) \rho_{n 1}(-k)+\rho_{n 2}(-k) \rho_{n 2}(k)\right] \\
& =\sum_{n=1}^{N} \frac{v_{F n}}{2} \int d x\left[\left(\partial_{x} \phi_{n}(x)\right)^{2}+P_{n}(x)^{2}\right]
\end{aligned}
$$


where we have introduced the Fourier transforms: $\rho(k)=$ $\int d x e^{i k x} \rho(x)$, and the field operator $\phi$ and its conjugated momentum $P$ given by

$$
\rho_{1}(x)+\rho_{2}(x)=\frac{1}{\sqrt{\pi}} \partial_{x} \phi, \quad \rho_{1}(x)-\rho_{2}(x)=-\frac{1}{\sqrt{\pi}} P .
$$

These fields obey the commutation rule $\left[\phi(x), P\left(x^{\prime}\right)\right]=$ $i \delta\left(x-x^{\prime}\right)$. The momentum $P$ is the difference between left and right movers and thus equivalent to the current operator.

We must furthermore find the bare charge difference in the boson language of the Luttinger model. Again modeling the interface between dot and lead as an abrupt barrier, we write the number of excess electrons, $q_{1}$, in the dot as

$$
\begin{aligned}
q_{1} & =\sum_{n=1}^{N} \int d x \Theta(x)\left[\rho_{n 1}(x)+\rho_{n 2}(x)\right] \\
& =-\frac{1}{\sqrt{\pi}} \sum_{n=1}^{N} \phi_{n}(0)
\end{aligned}
$$

for contact 1. The expression (18) is valid to leading order in $k / k_{F}$ for the Tomonaga model.

The barrier term splits into two terms when the electrons are divided into the left and right mover sections: a forward and backward scattering part,

$$
H_{\mathrm{barr}}=\sum_{n=1}^{N} \frac{V_{n}}{L} \sum_{k i} \rho_{n k i}+\sum_{n=1}^{N} \frac{V_{n}}{L} \sum_{k k^{\prime}}\left(c_{n k 1}^{\dagger} c_{n k^{\prime} 2}+\text { H.c. }\right) \text {. }
$$

The forward scattering term can be absorbed as a phase shift and is not important. The backscattering term cannot be expressed as a bilinear form and in order to deal with this term, we must use the boson representation of the Fermi operators. We will use a formulation due to Schotte and Schotte ${ }^{24}$ for the $\mathrm{x}$-ray edge problem. The bosonization technique was later generalized. ${ }^{25}$ The reason for using the first is that in the present problem we need only the boson form of the local electron operators at $x=0$. Now define the fermion operators

$$
C_{n i}=\frac{1}{\sqrt{N_{L}}} \sum_{k} c_{n k i}
$$

where $N_{L}$ is the number of states in our normalization volume: $N_{L}=\sum_{k} 1$. We have therefore introduced an upper cutoff $k_{D}$, that is given by the bandwidth: $k_{D}=$ $2 \pi N_{L} / L$. The new local operators obey the usual anticommutation relations,

$$
\begin{aligned}
& \left\{C_{n i}^{\dagger}, C_{n^{\prime} i^{\prime}}\right\}=\delta_{n n^{\prime}} \delta_{i i^{\prime}} \\
& \left\{C_{n i}, C_{n^{\prime} i^{\prime}}\right\}=0
\end{aligned}
$$

Following Schotte and Schotte we note that $\left[\rho(k), C^{\dagger}\right]=$ $C^{\dagger}$, which suggests that we can write

$$
C_{n 1,2}^{\dagger}=\frac{1}{\sqrt{2}} \exp \left( \pm \frac{2 \pi}{L} \sum_{k>0} \frac{1}{k}\left[\rho_{n 1,2}(k)-\rho_{n 1,2}(-k)\right]\right)
$$

where the prefactor is fixed so that the commutation relations (21) are fulfilled for $n=n^{\prime}$ and $i=i^{\prime}$. However, for different mode numbers and for the two different species of electrons, anticommutations relations must also be maintained, and it is necessary to include an extra phase factor in the bosonized form of the $C$ 's. Each $C_{n i}$ should have an extra factor: $\exp \left(i \theta_{n i}\right)$ defined $\operatorname{as}^{25}$ (Jordan-Wigner transformation)

$$
\theta_{n 1}=\pi \sum_{j=1}^{n-1} N_{j 1}, \quad \theta_{n 2}=\pi N_{1}+\pi \sum_{j=1}^{n-1} N_{j 2}
$$

where we have defined the number operators $N_{n i}$ corresponding to the operators $C_{n i}$. Fortunately, we can show that this complication can be disregarded. First we observe that the $N$ 's commute with the remaining part of the Hamiltonian. If we then consider, e.g., the partition function and expand it in orders of the perturbation it can be shown that the operators can always be rearranged so that the phase factors disappear. Therefore, they can be omitted.

The backscattering term can now be written in terms of the boson operator by the use of Eq. (22), and we finally arrive at the fully bosonized form of the model Hamiltonian,

$$
\begin{aligned}
H= & H_{10}+H_{1 \mathrm{el}}+\frac{U_{D}}{\pi}\left(\sum_{n=1}^{N_{1}} \phi_{1 n}(0)-\pi q_{2}\right)^{2} \\
& +\sum_{n=1}^{N_{1}} \frac{V_{n}}{2 a_{n}}\left[\exp \left(2 i \sqrt{\pi} \phi_{1 n}(0)\right)+\text { H.c. }\right]
\end{aligned}
$$

where $a_{n}=1 / 2 \pi k_{D n}$ defines the inverse upper cutoff for $k$. Here we have only performed the bosonization for the degrees of freedom (denoted by $\phi_{1 n}$ ) corresponding to charge transfer through contact 1 , which is relevant for the calculation of the conductance in the $G_{2} \ll G_{H}$ case discussed in Sec. IV A, whereas in the calculation in Sec. IV B both contacts are dealt with in the boson representation.

The exponential in Eq. (24) is a displacement operator which displaces the variable conjugated to $\sqrt{\pi} \phi_{n}(0)$ [which is $P(0)$ ] by 2 . The factor of 2 appears because the backscattering term turns a forward moving particle into a backward moving particle, giving a total current change of 2 .

\section{B. Effective action involving only the local fields}

The model Hamiltonian has now been transformed into a series of coupled Luttinger liquids. The next step in our calculation is to integrate out all degrees of freedom except those associated with the $\phi_{n}(0)$ 's. This can be done because the nonquadratic terms involve only $\phi_{n}(0)$. Consider now an imaginary time Feynman path-integral 
form of the bosonized model above. After the integration, which is done in Appendix A, the part of the action corresponding to $H_{0}$ reads

$$
S_{i 0}=\sum_{n=1}^{N_{i}} \frac{1}{\beta} \sum_{\omega}|\omega|\left|\phi_{i n}(i \omega)\right|^{2}
$$

where $i$ is 1 or 2 and $\phi$ denotes $\phi(x=0)$. The $\omega$ sum is for each $n$ restricted to energies smaller than a cutoff: $\omega_{C n}=\pi v_{F n} / a_{n}$; see Appendix A for a discussion.

The effective action for the local fields, $\phi_{n}$, corresponding to the Hamiltonian in Eq. (24) then takes the form

$$
\begin{aligned}
S= & S_{10}+\frac{U_{D}}{\pi} \frac{1}{\beta} \sum_{\omega}\left|\sum_{n=1}^{N_{1}} \phi_{1 n}-\pi q_{2}\right|^{2} \\
& +\sum_{n=1}^{N_{1}} \frac{V_{n}}{a_{n}} \int_{0}^{\beta} d \tau \cos \left(2 \sqrt{\pi} \phi_{1 n}\right),
\end{aligned}
$$

which completes the bosonization part. In the following the action above is used to derive the temperature dependent conductance.

\section{THE CONDUCTANCE OF THE QD DEVICE}

We shall here calculate the conductance using perturbation theory in the weak and strong coupling limits, i.e., for a weak tunneling matrix element and weak scattering barrier, respectively. Contact number 1 is assumed to be strongly coupled to the lead, whereas contact 2 is considered in both of the two limits.

\section{A. The conductance for $G_{2} \ll G_{H}$}

For the case where the nominal conductance of contact 2 is small the expression for current through the QD is given by Eq. (6). The spectral function $P(\omega)$ must be calculated with respect to the action in Eq. (26) (setting $\left.q_{2}=0\right)$. To zeroth order in $V_{n}$ we obtain

$$
P^{(0)}(t)=\exp \left(\frac{2}{N_{1}} \int_{-\infty}^{\infty} \frac{d \epsilon}{\epsilon} \frac{\left(e^{-i \omega t}-1\right)\left[1+n_{B}(\omega)\right]}{1+\left[\pi \omega / N_{1} U\right]^{2}}\right) .
$$

Comparing with the theory of a single junction,,$^{9-11}$ we see that the characteristic of a QD biased so that one QPC is in the tunneling regime, while the second has $N$ fully transmitting channels, equivalent to a single junction seeing an environment impedance of $Z=h /\left(N_{1} e^{2}\right)$. The low energy behavior of $P^{(0)}(\omega)$ is given by $P^{(0)}(\omega) \approx$ $\left(\omega / U_{D}\right)^{2 / N_{1}-1}$. At low energies the higher order terms in $V_{n}$ become relevant and we have calculated $P$ to second order in $V_{n}$. Details are offered in Appendix B. The low energy behavior is of the form

$$
P(\omega) \approx P^{(0)}(\omega)\left(1-\kappa\left(\omega / U_{D}\right)^{2 / N_{1}} \sum_{m=1}^{N_{1}} W_{1 m}\right),
$$

where $\kappa$ is a constant of order 1 and where the constants
$W_{1 m}=V_{1 m}^{2} / v_{F m}^{2}$ can be related to the reflection probabilities and thus also to the nominal conductance (see Sec. VI), $W_{1 n} \approx R_{1 n}$. Insertion of Eq. (28) into Eq. (6) leads to a low temperature conductance given by

$$
G(T) \approx G_{0}\left(\frac{k T}{U}\right)^{2 / N_{1}}\left[1-\kappa\left(\frac{k T}{U}\right)^{-2 / N_{1}} \sum_{m=1}^{N_{1}} R_{1 m}\right]
$$

The result in Eq. (29) shows that the perturbation theory is inadequate when the second term in the parentheses becomes of order 1 . The consequence of the increase in the correction term as, e.g., the temperature is lowered, will be discussed in Sec. VI.

\section{B. The conductance for $G_{2} \sim N_{2} G_{H}$}

Next we consider the situation where junction number 2 has a nominal conductance close to a quantized value and again do perturbation theory in the barrier. Contact 1 has a number, given by $N_{1}$, of fully transmitting channels. The conductance to linear order in the voltage across junction 2 can be calculated from the Kubo formula. For the case where $G_{2} \sim N_{2} G_{H}$ it is appropriate to use the boson representation for the $q_{2}$ degrees of freedom as well. In the boson language the current operator is given by

$$
I=e \dot{q}_{2}=-\frac{e}{\sqrt{\pi}} \sum_{n=1}^{N_{2}} \dot{\phi}_{2 n}
$$

The frequency dependent conductance is then given by the usual Kubo formula,

$$
G(\omega)=\frac{1}{\omega} \operatorname{Im} \Pi(\omega+i \delta),
$$

where $\Pi$ is the current-current correlation function. If we denote $\Phi_{2} \equiv \sum_{n=1}^{N_{2}} \phi_{2 n}$, the Matsubara current-current correlation function becomes

$$
\Pi(i \omega)=\frac{e^{2} \omega^{2}}{\pi \beta}\left\langle\left|\Phi_{2}(i \omega)\right|^{2}\right\rangle
$$

The expectation value should be taken with respect to an action where both contacts are treated in the boson representation. Since contact 1 is assumed to have $N_{1}$ channels with negligible reflection, these can be integrated out and we are left with an effective action involving the $\phi_{2}$ fields,

$$
\begin{aligned}
S= & S_{20}+\frac{1}{\beta} \sum_{\omega} H(i \omega)\left|\Phi_{2}(i \omega)\right|^{2} \\
& +\sum_{n=1}^{N_{2}} \frac{V_{2 n}}{a_{n}} \int_{0}^{\beta} d \tau \cos \left(2 \sqrt{\pi} \phi_{2 n}\right),
\end{aligned}
$$

where

$$
H(i \omega)=|\omega| \frac{1}{N_{1}+\pi|\omega| / U_{D}}
$$


First we find the zeroth order expression for the conductance. In this case the action can be written in terms of $\Phi_{2}$ alone. We obtain

$$
S^{(0)}=\frac{1}{\beta} \sum_{\omega}\left|\Phi_{2}(i \omega)\right|^{2} D^{-1}(i \omega),
$$

where

$$
D^{-1}(i \omega)=\left(H(i \omega)+\frac{|\omega|}{N_{2}}\right)
$$

and the current-current correlation function follows as

$$
\Pi(i \omega)=\frac{e^{2} \omega^{2}}{2 \pi} D(i \omega)
$$

Inserting this into Eq. (32), we find for the conductance for $V_{n}=0$,

$$
G^{(0)}(\omega)=N_{2} \frac{e^{2}}{h} \operatorname{Re} \frac{1}{1+\frac{N_{2}}{N_{1}+i \omega \pi / U_{D}}} .
$$

The low frequency result is a constant nonzero conductance,

$$
G^{(0)}(0)=\frac{e^{2}}{h} \frac{N_{2} N_{1}}{N_{1}+N_{2}},
$$

corresponding to a series coupling of the two QPC's. This does not, however, mean that the conductance is finite at zero temperature because the next leading term in $V_{2 n}$ gives a divergent correction at low frequencies.

As was discussed above, the present calculation is equivalent to a single junction connected to an environment with impedance given by $1 / N_{1} G_{H}$. Some calculations ${ }^{14,17,26}$ where higher order tunneling events were considered have ignored the discharge of the tunnel junction capacitor after a tunnel event. This corresponds to an infinitely large impedance or $N_{1}=0$, in which case we have for the frequency dependent conductance,

$$
G^{(0)}(\omega)=\frac{e^{2}}{h} \frac{N_{2} \omega^{2}}{\omega^{2}+\left(U N_{2} / \pi\right)^{2}} .
$$

For small frequencies the conductance goes to zero as $\left(\omega / U_{D}\right)^{2}$ in agreement with the findings in Ref. 26, where this result was found for a single channel model regardless of the value of the tunneling matrix element, and with perturbation theory to second order in the tunneling matrix element. ${ }^{27}$ Zwerger and Scharpf found that the conductance decreases quadratically with temperature for this model. The result in Eq. (40) shows that a model which ignores the discharging effects cannot have a finite dc conductance even in this extreme case where some channels are perfectly transmitting.

To next leading order in $V_{n}$, the calculation is somewhat more complicated and again details are given in Appendix B. We find for the low temperature and zero frequency result,

$G(k T)-G^{(0)} \approx-\frac{e^{2}}{h}\left(\frac{k T}{U_{D}}\right)^{-2 /\left(N_{1}+N_{2}\right)} \sum_{n=1}^{N_{2}} R_{2 n}$.
The correction to the conductance is seen to diverge at small energies in agreement with the renormalizationgroup arguments presented in the following section. However, the divergence is slower than for the case studied in Sec. IV A where only one junction had channels with transmission probability of order 1 .

\section{RENORMALIZATION-GROUP ANALYSIS}

The effective action cannot be solved in general. However, some conclusions can be drawn from renormalization-group arguments. We will consider the scaling behavior of the effective action in the limit of small barriers and use arguments similar to those employed by Fisher and Zwerger, ${ }^{28}$ which can be carried over directly. The strategy is to integrate out the high energies and then rescale so that the Hamiltonian keeps its form.

The two configurations studied in Sec. IV correspond to different physical situations: The junction with channels close to pinchoff sees either a closed (Sec. IV A) or an opened (Sec. IV B) second contact. Here we consider the scaling behavior of contact 2 having a small reflection probability, while contact 1 has $N_{1}$ open channels. These may be easily integrated out. Further, in order to perform the perturbative renormalization-group analysis of the channels with transmission close to 1 , we should take all the $N_{2}-1$ other barriers to zero and integrate out the corresponding channels. This will lead to an effective action for the channel in question. The algebra is thus straightforward and we just quote the result. We get an effective low-energy action given by

$$
S_{n}=\frac{1}{g \beta} \sum_{\omega}|\omega|\left|\phi_{2 n}(i \omega)\right|^{2}+\frac{V_{2 n}}{2 a_{n}} \int d \tau \cos \left[2 \sqrt{\pi} \phi_{2 n}(\tau)\right]
$$

where

$$
g=1-\frac{1}{N_{1}+N_{2}} .
$$

The effective action thus reduces to the model considered by Kane and Fisher. ${ }^{19}$ These authors study a one channel Luttinger model with a single barrier. In the Luttinger model the coupling constant $g$ is related to the interaction, being larger than 1 for attractive actions and smaller than 1 for repulsive interactions. The renormalization group has been considered by several authors. ${ }^{29,30,28}$ The barrier parameter can be shown to obey the scaling equation,

$$
\frac{d V_{2 n}}{d l}=(1-g) V_{2 n}
$$

Since $g<1$ we can see that $V_{n}$ will crow under scaling and the barrier is always a relevant perturbation. The scaling equation is valid only to lowest order in $V_{2 n}$. However, Fisher and Zwerger ${ }^{28}$ argued that it is true to all orders since higher order terms generate only local terms whereas the "friction term" is nonlocal in time and similar arguments can here be applied to the cross terms with different $V_{n}$ 's. This conclusion can be supported by 
comparing with the opposite representation where the junction is viewed as a tunnel junction. In this case the tunnel matrix element can be seen always to be irrelevant to lowest order since the second order result for the conductance vanishes as $T \rightarrow 0$. The tunneling conductance follows the power law: $G \sim T^{2 / N_{1}}$ or decreases exponentially for $N_{1}=0$.

\section{CROSSOVER TO COULOMB BLOCKADE BEHAVIOR: RENORMALIZED CAPACITANCE OF THE QD}

The scaling equations derived above give the temperature dependence of the renormalized barriers. The scaling procedure has to be stopped when the high energy cutoff hits $k T$. From the scaling equation (44), we get the renormalized barriers

$$
W_{2 n}(k T) \sim W_{2 n}\left(U_{D}\right)\left(\frac{k T}{U_{D}}\right)^{-2 /\left(N_{1}+N_{2}\right)},
$$

in agreement with the perturbation theory results in Sec. IV. The coefficients $W_{2 n}$ can be related to the nominal conductance of the contact. For a $\delta$-function potential model used here, we have that

$$
G_{2}=G_{H} \sum_{n=1}^{N_{2}} \frac{1}{1+W_{2 n}} ; \quad W_{2 n}=V_{2 n}^{2} / v_{F n}^{2}
$$

and for small reflection probability, $R_{n}$, we have $W_{n}=$ $R_{n}$. The perturbative renormalization-group approach breaks down when $R(k T)$ becomes of order of 1 . At this point it is more appropriate to represent the junction as a weak link instead of as a weak barrier. The crossover temperature between these regimes is given by

$$
\frac{k T_{c}}{U_{D}} \approx R^{\left(N_{1}+N_{2}\right) / 2}=\left(1-\frac{G_{2}}{N_{2} G_{H}}\right)^{\left(N_{1}+N_{2}\right) / 2}
$$

For energies less than $T_{c}$ we represent the contact as a tunnel junction with the usual results for the conductance. However, the energy scale that enters the temperature dependence (or voltage dependence) of the conductances is given by $T_{c}$ and may thus be strongly renormalized. These findings are summarized in Table I.

It is interesting to note that in the case of a large number of channels a similar crossover temperature has been found. Odintsov ${ }^{14}$ and Zwerger and Scharpf ${ }^{17}$ found that for a single junction with a large nominal conductance the Coulomb blockade behavior exist for temperatures less than $U_{D} \exp \left(-G_{0} / 2 G_{H}\right)$.

We can interpret the crossover temperature in Eq. (47) as the renormalized charging energy, because it is the charging energy that would be determined from the experimental characteristics. The effective capacitance is by this definition given by

$$
C^{*}=C_{0}\left(1-T_{2}\right)^{-\left(N_{1}+N_{2}\right) / 2} .
$$

Recent experiments ${ }^{4}$ on conductance through a quantum dot have shown that the Coulomb oscillations vanish as the conductance of one of the point contacts is increased. Similar effect has been observed by Foxman et $a .^{5}$ in an applied magnetic field. The present theory explains these experimental observations.

\section{DISCUSSION}

We have established an analogy between tunnel junctions in the Coulomb blockade regime and a Luttingertype model. The formalism introduced here allows us to calculate the behavior for an arbitrary number of connecting channels. In particular, we have studied the case when a QD is strongly coupled to the leads through QPC's with nearly perfectly transmitted modes. For that situation it was found that there is a high temperature regime where the conductance decreases with a weaker power law than in the low temperatures regime where Coulomb blockade behavior is found. We have found the crossover temperature between the two regimes.

The model calculation for junctions with only a few channels explains the observed vanishing of the charging energy as the transmission probability approaches 1 . We have argued that the observed decreasing charging energy can be understood in terms of a renormalized capacitance due to the smearing of the charge on the dot by quantum charge fluctuations through the contacts. The effective capacitance, defined by the crossover temperature where the Coulomb blockade effect sets in, leads to a relationship between the transmission probability of the junction and the charging energy as measured from the temperature dependence. We believe that it should be possible to experimentally verify the predicted dependence of the number of channels and the predicted dependence of the crossover temperature on the nominal conductance.

It should be noted that it is the capacitance as mea-

TABLE I. Summary of the findings for the temperature dependence of conductance of the QD device shown in Fig. 1 for the case when one or both of the junctions have channels with transmission probability close to unity.

\begin{tabular}{lcc}
\hline \hline & $G_{2} \ll G_{H}$ and $G_{1} \sim N_{1} G_{H}$ & $G_{2} \sim N_{2} G_{H}$ and $G_{1}=N_{1} G_{H}$ \\
\hline$T_{c} / U_{D}$ & $R_{1}^{2 / N_{1}}$ & $R_{2}^{2 /\left(N_{1}+N_{2}\right)}$ \\
$T>T_{c}$ & $G \sim G_{0}\left(T / U_{D}\right)^{2 / N_{1}}$ & $G-G_{0} \sim-R_{2}\left(T / U_{D}\right)^{-2 /\left(N_{1}+N_{2}\right)}$ \\
$T<T_{c}$ & $G \sim G\left(T_{c}\right) \exp \left(-T / T_{c}\right)$ & $G \sim G\left(T_{c}\right)\left(T / T_{c}\right)^{2 / N_{1}}$ \\
\hline \hline
\end{tabular}


sured from the transport properties that we have calculated. The capacitance that enters in the period of the Coulomb oscillations is given by the bare capacitances. The period is determined solely by the electrostatic energy degeneracy of the state with $N_{D}$ electrons on the dot and the state with $N_{D}+1$ electrons.

We also studied the situation when one contact has a number of fully transmitting channels and the second contact is in the tunneling regime. Within the present model, it was shown to be equivalent to a single junction in the Coulomb blockade regime coupled to an environment with impedance $h / e^{2}$ divided by the number of transmitting channels.

\section{ACKNOWLEDGMENTS}

The author acknowledges valuable discussions with P. Hedegård, A.-P. Jauho, M. Jonson, J. Kinaret, A. Luther, A. Nersesyan, and R. Shekhter.

\section{APPENDIX A: INTEGRATING OUT THE NONLOCAL DEGREES OF FREEDOM}

The integration over the $\phi(x \neq 0)$ degrees of freedom used in Sec. III is here discussed in more detail. The first step is done by introducing an auxiliary degree of freedom through the functional $\delta$ functions,

$$
\delta\left[\phi_{n}(x=0, \tau)-\theta_{n}(\tau)\right]=\mathcal{N} \int \mathcal{D}\left[\lambda_{n} / 2 \pi\right] \exp \left(i \int_{0}^{\beta} d \tau \lambda_{n}(\tau)\left[\phi_{n}(x=0, \tau)-\theta_{n}(\tau)\right]\right)
$$

Here $\mathcal{N}$ is a normalization constant. This allows us to replace the $\phi_{n}(x=0)$ appearing in the action with $\theta_{n}$, if we also include a functional integral over the $\theta_{n}$ 's. The action corresponding to $H_{0}$ plus that coming from the $\delta$ function is then

$$
\begin{aligned}
S_{0}= & \frac{1}{2} \sum_{n=1}^{N} \int_{0}^{\beta} d \tau \int d x\left(\frac{1}{v_{\mathrm{F} n}} \dot{\phi}_{n}^{2}+v_{F n}\left(\partial_{x} \phi_{n}\right)^{2}\right) \\
& +i \sum_{n=1}^{N} \int_{0}^{\beta} d \tau \lambda_{n}\left[\phi_{n}(x=0, \tau)-\theta_{n}(\tau)\right],
\end{aligned}
$$

which after integration over $\phi(x)$ and $\lambda$ becomes

$$
S_{0}=\frac{1}{\beta} \sum_{\omega} \sum_{n=1}^{N}|\omega|\left|\theta_{n}(i \omega)\right|^{2}
$$

where $i \omega$ is the Matsubara frequencies.

Note that the Fermi velocities have dropped out due to the infinite bandwidth approximation. However, we need to reintroduce the energy scale given by the Fermi velocity through the cutoff energy in the above action. How this should be done can be seen, e.g., by considering the correlation function

$$
B(\tau)=\frac{1}{L} \sum_{k k^{\prime} q q^{\prime}}\left\langle\mathcal{T}\left(c_{k 1}^{\dagger} c_{k^{\prime} 2}\right)(\tau)\left(c_{q 1}^{\dagger} c_{q^{\prime} 2}\right)(0)\right\rangle_{0}
$$

where the average is taken with respect to the unperturbed electronic part of the Hamiltonian. Using the original Hamiltonian, we get at $T=0$

$$
B(\tau)=\frac{1}{\left(2 \pi v_{F} \tau\right)^{2}}
$$

whereas the bosonized form yields

$$
\begin{aligned}
B(\tau) & =\frac{1}{4 a^{2}}\left\langle e^{i 2 \sqrt{\pi} \phi(\tau)} e^{-i 2 \sqrt{\pi} \phi(0)}\right\rangle \\
& \simeq \frac{1}{4 a^{2}} \exp \left(\int_{0}^{\omega_{D}} \frac{d \omega}{\omega}[\cos (\omega \tau)-1]\right) .
\end{aligned}
$$

The integral in the exponent gives for large imaginary times $\simeq-2 \ln \left(\omega_{D} \tau\right)$, and hence we recover the correct result for the correlation function with $\omega_{D}$ defined as $\omega_{D}=\pi v_{\mathrm{F}} / a$.

\section{APPENDIX B: PERTURBATION THEORY IN THE SCATTERING BARRIERS}

Here we show in some detail how the perturbative results quoted in Sec. IV are reached. The object is to calculate the function $P$ defined in Eq. (7) to second order in $V_{n}$, and likewise for the current-current correlation function $\Pi$ defined in Eq. (32). In the former case the relevant action is given by (26) (with $q_{2}=0$ ) whereas for the calculation of the current-current correlation function we should use the action in Eq. (33). The former action is identical to the latter if we put $N_{1}=0$ in the function $H$ and identify $\phi_{2}$ with $\phi$ and $N_{2}$ with $N_{1}$. We will work with (33) below.

If we add the following term to the action:

$$
S_{S}\left[\Phi_{2}, \xi\right]=\frac{1}{\beta} \sum_{\omega}\left\{\left[\Phi_{2}(i \omega)\right]^{*} \xi(i \omega)+\text { c.c. }\right\}
$$

and consider the functional defined by

$$
Z[\xi]=\left(\prod_{n} \int \mathcal{D}\left[\phi_{2 n}\right]\right) \exp \left(-S\left[\left\{\phi_{2 n}\right\}\right]-S_{S}[\Phi, \xi]\right)
$$

then the functions $P$ and $\Pi$ can be written as

$$
\begin{aligned}
P(\tau) & =Z[g(\tau)] / Z[0] \\
\Pi(\omega) & =\left.\frac{\beta \omega^{2}}{2 \pi} \frac{1}{Z[0]} \frac{d^{2} Z[\xi]}{d \xi(i \omega) d \xi^{*}(i \omega)}\right|_{\xi=0},
\end{aligned}
$$

where we have defined $g=U_{D}\left(e^{i \omega \tau}-1\right) / i \omega \sqrt{\pi}$ and used $\Phi_{2}=\sum_{n=1}^{N_{2}} \phi_{2 n}$.

We write the part of the action that couples the different channels as 


$$
\begin{aligned}
\exp \left[-\frac{1}{\beta} \sum_{\omega} H(i \omega)\left|\Phi_{2}(i \omega)\right|^{2}\right] \\
=\mathcal{N} \int \mathcal{D}[\lambda] \exp \left[-\frac{1}{\beta} \sum_{\omega} H^{-1}(i \omega)|\lambda(i \omega)|^{2}\right] \\
\times \exp \left[-\frac{i}{\beta} \sum_{\omega}\left[\Phi_{2}^{*}(i \omega) \lambda(i \omega)+\text { c.c }\right]\right]
\end{aligned}
$$

where $\mathcal{N}$ is a normalization constant. The functional $Z[\xi]$ is then expressed as

$$
Z[\xi]=\mathcal{N} \int \mathcal{D}[\lambda] \exp \left(-\frac{1}{\beta} \sum_{\omega} H^{-1}|\lambda|^{2}\right) F[\xi+i \lambda]
$$

where

$$
F[\xi+i \lambda]=\left(\prod_{n=1}^{N_{2}} \int \mathcal{D}\left[\phi_{2 n}\right]\right) \exp \left(-S_{0}\left[\phi_{2 n}\right]\right) \exp \left(-\frac{1}{\beta} \sum_{\omega} \sum_{n=1}^{N_{2}}\left\{\phi_{2 n}(i \omega)[\xi(i \omega)+i \lambda(i \omega)]+\text { c.c. }\right\}\right)
$$

Here we have defined $S_{0}$ as the diagonal parts of the total action $S$.

The functional $F$ is easy to obtain to lowest order in the scattering term. After integration over the $\phi$ fields, we simply get

$F^{(0)}[\lambda+\xi]=\mathcal{N}_{0}^{-1} \exp \left(\sum_{n=1}^{N_{2}} \frac{1}{\beta} \sum_{\omega} \frac{\left(\xi^{*}+i \lambda^{*}\right)(\xi+i \lambda)}{|\omega|}\right)$,

where $\mathcal{N}_{0}^{-1}=\prod_{n} \int \mathcal{D}\left[\phi_{2 n}\right] \exp \left(-S_{n}\left[\phi_{2 n}\right]\right)$. Inserting this into the expression for $Z$ and performing the $\lambda$ integration, we obtain

$$
Z^{(0)}[\xi]=\mathcal{N}_{0}^{-1} \exp \left(\frac{1}{\beta} \sum_{\omega} D(i \omega)|\xi(i \omega)|^{2}\right)
$$

By the use of Eq. (B7), we now get the result for the conductance to zeroth order which is also obtained in Sec. IV.

The next leading order expression is found by expanding $F$ to second order in $V_{2 n}$,

$$
\begin{aligned}
F^{(2)}[\lambda+\xi]= & \left(\prod_{n=1}^{N_{2}} \int \mathcal{D}\left[\phi_{2 n}\right]\right) \exp \left(-S_{0}\left[\phi_{2 n}\right]\right) \exp \left(-\frac{i}{\beta} \sum_{\omega} \sum_{n=1}^{N_{2}}\left[\phi_{2 n}(\lambda+\xi)+\text { c.c. }\right]\right) \\
& \times \sum_{m=1}^{N_{2}}\left(\frac{V_{2 m}}{2 a_{m}}\right)^{2} \int_{0}^{\beta} d \tau \int_{0}^{\beta} d \tau^{\prime} \cos \left\{2 i \sqrt{\pi}\left[\phi_{m}(\tau)-\phi_{m}\left(\tau^{\prime}\right)\right]\right\} .
\end{aligned}
$$

Performing the integration over the $\phi$ fields and inserting the resulting functional into Eq. (B6) leads to the second order result for the generating functional,

$$
\begin{aligned}
Z^{(2)}[\xi]=Z^{(0)}[\xi] \sum_{m=1}^{N_{2}} \int_{0}^{\beta} d \tau \int_{0}^{\beta} d \tau^{\prime} \sum_{m=1}^{N_{2}} \alpha_{m}\left(\tau-\tau^{\prime}\right) & \times \exp \left(\frac{i \sqrt{\pi}}{\beta} \sum_{\omega}\left[\frac{1}{|\omega|+N_{2} H(i \omega)} \xi(i \omega) f(i \omega)+\text { c.c. }\right]\right) \\
& \times \exp \left(\frac{\pi}{\beta} \sum_{\omega} \frac{H(i \omega)}{|\omega|\left[|\omega|+N_{2} H(i \omega)\right]}|f(i \omega)|^{2}\right),
\end{aligned}
$$

where $f=\exp (-i \omega \tau)-\exp \left(-i \omega \tau^{\prime}\right)$, and

$$
\alpha_{m}\left(\tau-\tau^{\prime}\right)=\left(\frac{V_{2 m}}{2 a_{m}}\right)^{2} \exp \left(-\frac{\pi}{\beta} \sum_{\omega}|f(i \omega)|^{2} /|\omega|\right) .
$$

This function is by definition given by the function $B(\tau)$ defined in Eq. (A4), and we have that

$$
\alpha_{m}(\tau)=W_{2 m} \int_{-\infty}^{\infty} d \omega \omega \frac{e^{\omega|\tau|}}{e^{\beta \omega}-1} .
$$

We can now compute the current-current correlation function following Eq. (B7) and subsequently obtain the conductance to second order in $W$ from analytic continuation of $\Pi^{(2)}(i \omega)$. After some algebra, we obtain 


$$
\begin{array}{r}
G^{(2)}(\omega)=\frac{e^{2}}{\omega}\left(\sum_{m=1}^{N_{2}} W_{2 m}\right)\left(\frac{N_{1}^{2}+\left(\pi \omega / U_{D}\right)^{2}}{\left(N_{1}+N_{2}\right)^{2}+\left(\pi \omega / U_{D}\right)^{2}}\right) \times \int_{-\infty}^{\infty} d t \int_{-\infty}^{\infty} d E e^{-i E t} B(t) \\
\times\left[(E-\omega) n_{B}(E-\omega)-(E+\omega) n_{B}(E+\omega)\right]
\end{array}
$$

where

$$
B(t)=\exp \left(-\frac{2}{\left(N_{1}+N_{2}\right)} \int_{-\infty}^{\infty} d \epsilon \frac{\left(e^{-i \epsilon t}-1\right)\left[1+n_{B}(\epsilon)\right]}{\epsilon\left\{1+\left[\pi \epsilon / U_{D}\left(N_{1}+N_{2}\right)\right]^{2}\right\}}\right) .
$$

To proceed further, we approximate the integral in the exponent above in Eq. (B15) by replacing the Lorentzian function with an exponential cutoff in which case we obtain

$$
B(t) \approx\left(1+\frac{i t(2 \gamma+N) U}{\pi}\right)^{2 /(2 \gamma+N)}
$$

at zero temperature. From this we can now show that at low temperatures and frequencies we get the correction to the conductance quoted in Eq. (41).

The result for the function $P$ can also be found from Eq. (B11). To second order we have

$$
P^{(2)}(\tau)=\sum_{n=1}^{N_{1}} \int_{0}^{\beta} d \sigma \int_{0}^{\beta} d \sigma^{\prime} \alpha_{m}\left(\sigma-\sigma^{\prime}\right) L_{m}\left(\sigma-\sigma^{\prime}\right)\left[h\left(\tau, \sigma, \sigma^{\prime}\right)-1\right] P^{(0)}(\tau)
$$

where

$$
\begin{aligned}
L_{m}\left(\sigma-\sigma^{\prime}\right) & =\exp \left(\frac{2 \pi}{\beta N} \sum_{\omega} \frac{1-\cos \left(\omega\left[\sigma-\sigma^{\prime}\right]\right)}{|\omega|\left(1+|\omega| \pi /\left[N_{1} U_{D}\right]\right)}\right) \\
& =\exp \left(\frac{2}{N_{1}} \int_{-\infty}^{\infty} \frac{d \epsilon}{\epsilon} \frac{\left(1-e^{-\epsilon\left|\sigma-\sigma^{\prime}\right|}\right)\left[1+n_{B}(\epsilon)\right]}{\epsilon\left(1+\left[\pi \epsilon / N_{1} U_{D}\right]^{2}\right)}\right) \\
& \approx\left(1+\frac{\left|\sigma-\sigma^{\prime}\right| U_{D} N_{1}}{\pi}\right)^{2 / N_{1}}, \\
h\left(\tau, \sigma, \sigma^{\prime}\right) & =\cos \left(\frac{2 \pi}{\beta N} \sum_{\omega} \frac{\cos (\omega[\tau-\sigma])-\cos \left(\omega\left[\tau-\sigma^{\prime}\right]\right)+\cos \left(\omega\left[\sigma^{\prime}\right]\right)-\cos (\omega[\sigma])}{|\omega|\left(1+|\omega| \pi /\left[U_{D} N_{1}\right]\right)}\right),
\end{aligned}
$$

and where $P^{(0)}$ were defined in Eq. (27). It is now straightforward to see that for large times $\tau U_{D} \gg 1$ we get that

$$
P^{(2)}(\tau) \sim\left(\sum_{m=1}^{N_{1}} W_{1 m}\right) P^{(0)}(\tau)\left(\tau U_{D}\right)^{2 / N_{1}}
$$

from which we can find the approximative form of $P(\omega)$ for small energies.

${ }^{1}$ R. Landauer, Philos. Mag. 21, 863 (1970).

${ }^{2}$ For a review, see D. V. Averin and K. K. Likharev, in Mesoscopic Phenomena in Solids, edited by B. L. Althuler, P. A. Lee, and R. A. Webb (Elsevier, Amsterdam, 1990).

${ }^{3}$ U. Meirav, M. A. Kastner, and S. J. Wind, Phys. Rev. Lett. 58, 771 (1990).

${ }^{4}$ L. P. Kouwenhoven et al., Z. Phys. B 85, 367 (1991).

${ }^{5}$ E. B. Foxman et al. (unpublished).

${ }^{6}$ A. T. Johnson et al., Phys. Rev. Lett. 69, 1592 (1992).

${ }^{7}$ B. W. Alpenaar et al., Phys. Rev. B 46, 7236 (1992).

${ }^{8}$ C. Pasquier et al., Phys. Rev. Lett. 70, 69 (1993).

${ }^{9}$ M. H. Devoret et al., Phys. Rev. Lett. 64, 1824 (1990).

${ }^{10}$ S. M. Girvin et al., Phys. Rev. Lett. 64, 3183 (1990).
${ }^{11}$ K. Flensberg et al., Phys. Scr. T42, 189 (1992).

${ }^{12}$ L. J. Geerligs, V. F. Anderegg, and C. A. van der Jeugd, Europhys. Lett. 10, 79 (1989).

${ }^{13}$ A. N. Cleland, J. M. Schmidt, and J. Clarke, Phys. Rev. Lett. 64, 1565 (1990).

${ }^{14}$ A. A. Odintsov, Zh. Eksp. Teor. Fiz. 94, 312 (1988) [Sov. Phys. JETP 67, 1265 (1988)].

${ }^{15}$ S. V. Panyukov and A. D. Zaikin, J. Low Temp. Phys. 73, 1 (1988).

${ }^{16}$ K. Flensberg and M. Jonson, Phys. Rev. B 43, 7586 (1991).

${ }^{17}$ W. Zwerger and M. Scharpf, Z. Phys. B 85, 421 (1991).

${ }^{18}$ S. V. Panyukov and A. D. Zaikin, Phys. Rev. Lett. 67, 3168 (1992). 
${ }^{19}$ C. L. Kane and M. P. A. Fisher, Phys. Rev. Lett. 68, 1220 (1992).

${ }^{20}$ L. I. Glazman, G. B. Lesovik, D. E. Khmel'nitskii, and R. I. Shekhter, Pis'ma Zh. Eksp. Teor. Fiz. 48, 218 (1988) [JETP Lett. 48, 238 (1988)].

${ }^{21}$ D. V. Averin and Y. V. Nazarov, Phys. Rev. Lett. 65, 2446 (1990).

22 J. Sólyom, Adv. Phys. 28, 201 (1970).

${ }^{23}$ G. D. Mahan, Many-Particle Physics (Plenum, New York, 1990).

${ }^{24}$ K. D. Schotte and U. Schotte, Phys. Rev. 182, 479 (1969).

${ }^{25}$ V. J. Emery, in Highly Conducting One-Dimensional Solids, edited by J. T. Devreese, R. P. Edwards, and V. E. van Doren (Plenum, New York, 1979).

${ }^{26}$ H. O. Frota and K. Flensberg, Phys. Rev. B 46, 15207 (1992).

${ }^{27}$ M. Ueda and F. Guinea, Z. Phys. B 85, 413 (1991).

${ }^{28}$ M. P. A. Fisher and W. Zwerger, Phys. Rev. B 32, 6190 (1985).

29 A. Schmid, Phys. Rev. Lett. 51, 1506 (1983).

${ }^{30}$ S. A. Bulgadaev, Pis'ma Zh. Eksp. Teor. Fiz. 39, 264 (1984) [JETP Lett. 39, 315 (1984)]. 